Bull. Mater. Sci., Vol. 20, No. 6, September 1997, pp. 901-908. (C) Printed in India.

\title{
Computer modelling of elastic stress effects during precipitation
}

\author{
T A ABINANDANAN \\ Centre for Advanced Study, Department of Metallurgy, Indian Institute of Science, \\ Bangalore 560 012, India
}

\begin{abstract}
We present a brief overview of computational studies of elastic stress effects during precipitation of coherent particles. These studies include those which employ a continuum model, an atomistic (microscopic) model, and a field model. Their relative merits and limitations are discussed. Also, elastic stress effects during precipitation are presented in order to highlight the capabilities of the different computational techniques used in these studies.
\end{abstract}

Keywords. Phase transformations; precipitation; elastic stress effects; microstructural evolution.

\section{Introduction}

During precipitation of a second phase in a solid system, elastic stresses may arise from particle-matrix misfit, compositional differences, interfacial stresses and external loads. These stresses may have a significant influence on the evolution of microstructure when the precipitating phase is coherent with the matrix. For example, stresses arising from misfit lead the particles to change their shape as they grow (Ardell et al 1966; Barnett et al 1974; Wang et al 1991a; Leo and Jou 1993; Thompson et al 1994; Abinandanan and Sankarasubramanian 1997), and to align themselves along specific crystallographic directions (Ardell 1970; Abinandanan 1991; Wang et al 1991b; Abinandanan and Johnson 1993). These behaviours can be altered further by the application of an external load on the system (Tien and Copley 1971; Miyazaki et al 1979; Hort and Johnson 1996). Since many technologically important alloys (such as superalloys, Ti-base alloys and Al-base alloys) exploit the presence of such coherent, misfitting particles for strengthening, an understanding of the stress effects on microstructure is essential both for processing (to produce a desirable microstructure) and for control during service (to ensure that the microstructure is stable).

In this paper, we give a brief overview of the modelling studies undertaken with the specific purpose of elucidating the effect of elastic stresses on microstructural evolution during precipitation. The rest of the paper discusses the salient features of continuum models $(\$ 2)$, atomistic simulations ( $\$ 3)$ and field models $(\$ 4)$. While some of the important results from these studies are highlighted, the emphasis is clearly on the models and computational techniques, so that the reader may have a broad view of the tools available for studying stressed solid systems.

\section{Continuum models}

The first systematic theoretical study of misfitting precipitates dates back to Eshelby's 
(1957, 1959) pioneering work on calculating the stress field around ellipsoidal particles assuming the particle and matrix phases to be elastic continua. There has been a large number of studies employing the 'energy approach', in which the strain energy is calculated for different particle configurations with a view to explain the experimentally observed microstructures (Miyazaki et al 1982; Khachaturyan et al 1988). The seventies saw the emergence of a thermodynamic formalism for treating coherent solids (Larche and Cahn 1973, 1978; Johnson and Alexander 1986; Leo and Sekerka 1989). This development made it possible to use a 'kinetic approach', in which precipitate growth is treated as due to solute acquisition at the precipitate-matrix boundary (see figure 1). For stressed solids, this approach requires a solution for the coupled elastic and diffusion fields in the two phases (Johnson 1986). Evidently, this approach is difficult-if not impossible-to use for studying the evolution of a microstructure with many particles, each with an arbitrary shape. Therefore, it is not surprising that only simple systems have been studied, as described in the next subsection. In all these studies, it is customary to neglect diffusion in the precipitate phase.

\subsection{Completely numerical methods}

The first class of studies take a completely numerical route for solving the differential

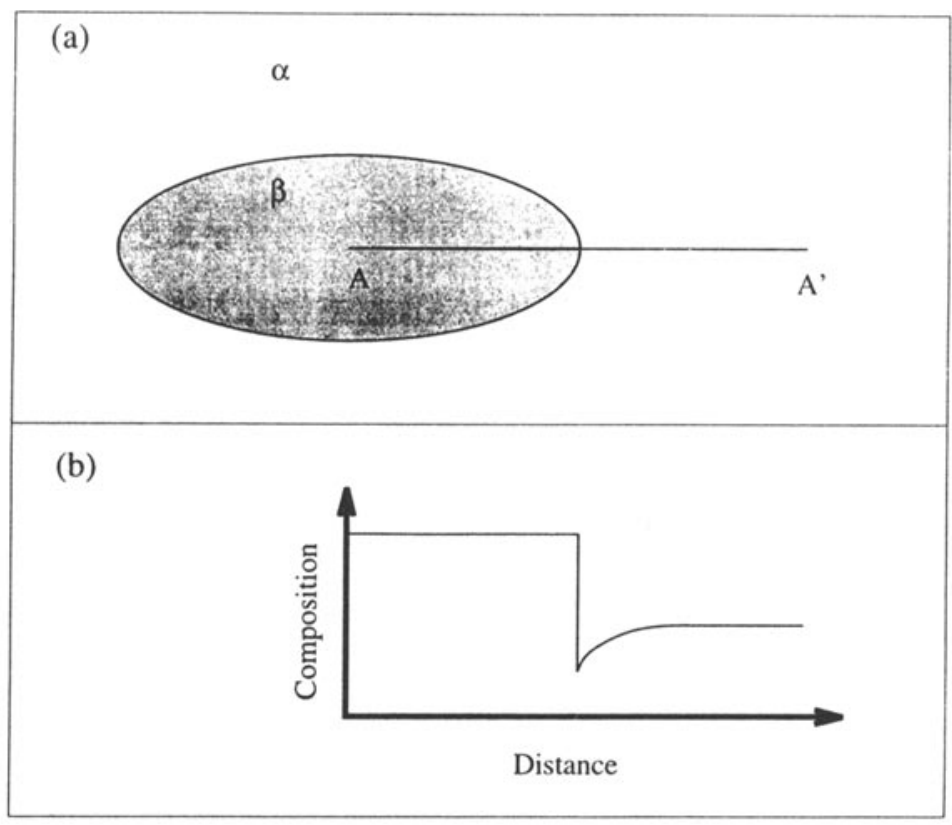

Figure 1. (a) Continuum models treat the two phases $\alpha$ and $\beta$ as elastic and diffusional continua, and precipitate growth is studied by solving for both elastic and diffusional fields simultaneously. (b) Composition field along the line $\mathrm{AA}^{\prime}$ in (a), indicating a jump in composition at the interface. The composition of the two phases at the interface are obtained using thermodynamics of stressed solids by assuming local equilibrium. These interfacial compositions serve as boundary conditions for solving the diffusion problem. 
equations. While this class could include the finite element (FEM), and the finite difference (FDM) methods, the boundary element method (BEM) has been the most widely employed (Voorhees et al 1988, 1992; Thompson et al 1994). These studies have examined shape evolution of a single precipitate (Thompson et al 1994), and the evolution of a small group of misfitting precipitates in two-dimensional (2-D) systems (Voorhees et al 1988, 1992). Due to the intensely numerical nature of the BEM, the systems which one can study are restricted to a small number of particles, and (at least so far) to 2-D systems. The formulation of BEM also restricts its use to elastically homogeneous systems (in which the precipitate and matrix phases have the same elastic constants). Though the use of FEM could overcome this difficulty, it is also numerically intensive, and the above mentioned restrictions to 2-D systems with small number of precipitates are likely to persist for some more time.

\subsection{Semianalytical methods}

Another group of studies have exploited the analytical, solutions to the elastic stress fields around precipitates of simple shapes, such as spheres or ellipsoids. For example, Abinandanan and Johnson (1993, 1995) have studied the coarsening behaviour of a collection of tetragonally misfitting, spherical particles. In their study, the system is isotropic and homogeneous; the isotropy allows an analytical solution to the elastic stress fields, while the homogeneity allows superposition of stress fields from different particles. They showed that while capillarity dominates the coarsening process during early stages (i.e. at small particle sizes), elastic stress effects gradually become progressively more dominant with increasing particle size, and cause particle clustering and alignment (Abinandanan and Johnson 1995), and retard the coarsening rate (Abinandanan 1991). More recently, Hort and Johnson (1996) extended this technique to examine an elastically inhomogeneous system with an externally applied uniaxial stress.

For elastically isotropic systems, stress fields around precipitates whose shapes are ellipsoids of revolution are available analytically up to a set of elliptic integrals (Mura 1987), which need to be solved numerically. These solutions have been exploited in a recent study by Abinandanan and Sankarasubramanian (1997) who examined the effect of difference in elastic constants on the shape evolution of an isolated, tetragonally misfitting precipitate. The precipitates are spherical at small sizes, and become progressively more ellipsoidal with increasing size. The rate at which the shape changes, however, was also found to be strongly dependent on difference in elastic constants of the two phases (Abinandanan and Sankarasubramanian 1997). It would be interesting to see if this large inhomogeneity effect is seen in other, more general (and more realistic) systems, such as those which have a hexagonal elastic anisotropy exhibited by the Ti-rich Ti-Al alloys with the $\alpha_{2}$ precipitates (Sankarasubramanian and Abinandanan, in preparation).

\subsection{Comments}

The continuum approach, whether it uses a fully numerical technique or a combination of analytical and numerical techniques, offers the advantage of studying the effect of different factors individually. For example, the role of elastic inhomogeneity 
and anisotropy in misfit and elastic constants could be studied separately, and in any combination. This is particularly useful, since it provides a rationale for choosing alloying additions specifically to exploit that factor which has the greatest influence on the microstructural evolution. On the other hand, the most important shortcoming of this approach is its reliance on numerical calculations for the elastic stress fields in any realistic system with anisotropic elastic properties, and with particles of non-ellipsoidal shapes, such as cuboids. Thus, any new development in analytical techniques in solving for the stress fields will greatly expand the use of this approach.

\section{Atomistic simulations}

Atomistic simulations of precipitation employ the alloy equivalent of the Ising model (see Gunton et al (1983) and Binder (1991) for recent reviews). In the simplest, binary alloy version of this model, atoms of the two species A and B occupy the sites on a rigid lattice, and interact with nearest neighbour atoms through pair-wise bonds of fixed energy $E_{\mathrm{AA}}, E_{\mathrm{BB}}$ and $E_{\mathrm{AB}}$. The model could be extended to include pair interactions among neighbours separated by larger distances, as well as multi-atom interactions through the so-called many-body potentials (Moulic et al 1994). The interactions among the atoms determine the nature of phase transformations in the system. For example, in the simple Ising model described above, when $E_{\mathrm{AB}}>\left(E_{\mathrm{AA}}-E_{\mathrm{BB}}\right) / 2$, the phase diagram exhibits a miscibility gap at low temperatures, and allows a study of spinodal decomposition, as well as precipitation of a B-rich phase from an A-rich matrix (Marro et al 1975).

\subsection{Thermodynamics: Spring models}

The assumption of rigid lattice in the conventional simulations does not allow the stress effects to be studied. In order to incorporate elastic stress effects, the model must allow atoms to be displaced from the lattice sites, and consider the interatomic interactions which depend continuously on the interatomic separation distance. A simple way of implementing these features in a model system is to introduce springs between neighbouring atoms; these springs lead to elastic interactions among the atoms (Fratzl and Penrose 1995; Desplat et al 1986). For example, Desplat et al (DBL) (1996) evaluated the thermodynamic properties of an Ising system in which the elastic interactions are included in the system Hamiltonian through a pair-wise energy term which is similar to that produced by a spring. While the elastic interactions in the DBL model are longitudinal, Fratzl and Penrose (FP) (1995) assume both longitudinal and transverse springs (see figure 2) which connect atoms up to the second neighbour shell. The transverse springs were introduced to overcome the limitation on the elastic moduli imposed by Cauchy's relation (Born and Huang 1954) for purely central forces. Both DBL and FP analyzed the effect of elastic interactions among atoms in a phase-separating system under a mean field approximation, and predicted a lowering of the coherent critical point in comparison with an unperturbed Ising model (Fratzl and Penrose 1996; Desplat 
et al 1996). In the DBL model, the miscibility gap was also shown to be asymmetric (Desplat et al 1996).

\subsection{Kinetics: Atom exchange mechanism}

While the above models have been studied from the point of view of the influence of elastic interactions on the phase diagram, FP have also extended their model to study the kinetics of phase transition (Fratzl and Penrose 1996). Such a study requires a mechanism for allowing the atoms to migrate, and FP chose a direct exchange mechanism, in which two unlike nearest neighbours are allowed to exchange their positions. However, it is well known that atomic transport in substitutional crystalline solids occurs through a vacancy mechanism, in which atoms exchange positions with neighbouring vacant sites. We note that realistic simulations employing the vacancy mechanism have been used on Ising systems with no elastic interactions (Athenes et al 1996; Abinandanan et al 1997). They are yet to be extended to include the elastic stress effects.

With a direct exchange model for atomic transport, FP also studied spinodal decomposition, and showed that the microstructure exhibits a strong anisotropy due

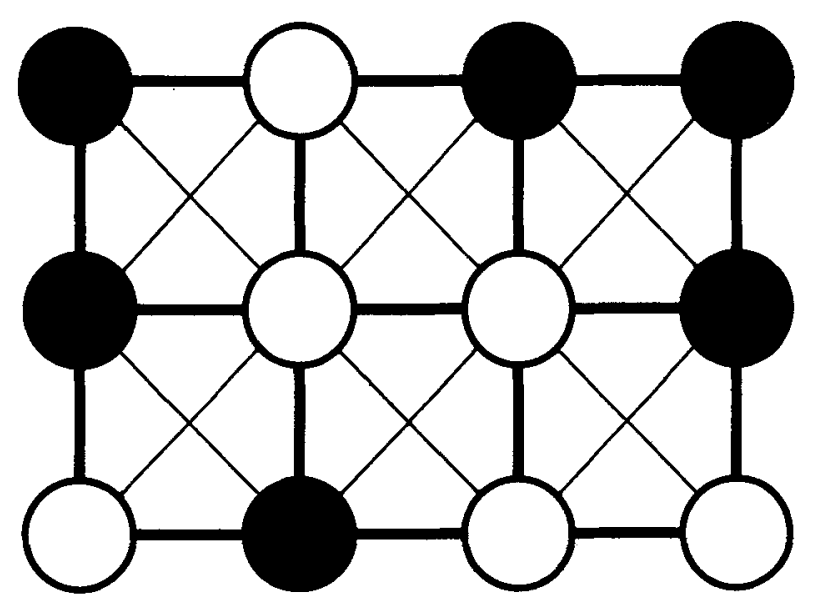

A

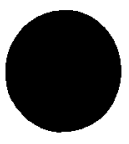

B

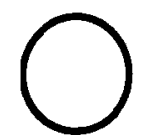

Figure 2. Schematic of the spring models: Atoms are assumed to be connected by springs (elastic bonds) represented by thick lines connecting nearest neighbours (nn), and thin lines connecting next nearest neighbours (nnn). Fratzl and Penrose (1995) consider both longitudinal springs between $\mathrm{nn}$ and $\mathrm{nnn}$, and transverse springs between $\mathrm{nn}$. The three spring constants are chosen so as to recover the macroscopic elastic constants in a cubic medium. Each spring constant has the same value irrespective of the identity of the two atoms connected by it. 
to an atomic size misfit; this anisotropy increased with time, misfit and composition, and decreased with temperature (Fratzl and Penrose 1996). The rate of coarsening was also found to be roughly independent of the misfit.

\subsection{Comments}

Due to the computationally intensive nature of atomistic simulations, the model systems are simple, and account for elastic interactions among atoms through the use of springs between atoms. Even with such a simple picture of an elastic solid, it has been possible to study only 2-D'systems, as in the study by Fratzl and Penrose (1996). Another significant limitation of such studies is the use of a direct exchange mechanism for atomic migration. As noted above, this is unrealistic. Thus, there exists a need for (i) a model which uses a vacancy mechanism, and (ii) efficient algorithms for computing the system energy at different configurations, so that realistic, three dimensional simulations can be performed.

\section{Field models}

\subsection{The approach and some key results}

Over the last few years, Khachaturyan and coworkers (1988) employed-a generalized phenomenological field approach to study the influence of elastic stress effects on precipitation (see the overview by Chen and Wang (1996) and references therein). In this approach, a free energy functional is written in terms of a set of spatially dependent field variables ('order parameters'), which in our case would include alloy composition and strain. Using a time-dependent Ginsburg-Landau formalism, a kinetic equation is derived for the temporal evolution of these field variables, and solved numerically. While we will not go into the details of this model, we recount some of the important conclusions drawn from their studies.

Wang et al (1991a) studied a 2-D system based on the cubic $\mathrm{Ni}-\mathrm{Al}$ system and showed a sequence of shape transitions which took an isolated particle from a circle to a square, which eventually split into a doublet (two parallel plates). While a circle $\rightarrow$ square transition has also been predicted by other studies based on a continuum model (Thompson et al 1994), the particle splitting, which arises from a shape instability, was observed for the first time in a simulation study. The particle shape instability, however, was observed to be initiated by the renucleation of the matrix phase at the centre of the particle; such an instability is yet to be observed experimentally. In systems with a larger number of particles, Wang et al (1991b) observed modulated ('rafted') microstructures in which particles align themselves along specific crystallographic directions in the crystal (Wang et al 1991b), an observation which is in qualitative agreement with experimental studies.

\subsection{Comments}

The phenomenological field approach of Khachaturyan and coworkers is an appealing new development. The kinetic equations have a firm basis in non-equilibrium 
thermodynamics, and once a model free energy functional is chosen, particle shape, alignment, etc are obtained directly from the simulations. A few words of caution, however, are in order. First, their use of mean field approximation for the system free energy, however, has been shown to lead to theoretically unsound predictions about thermodynamic instabilities (Abinandanan et al 1997). Moreover, their approach does not take into account the dynamics of fluctuations, due to their use of a mean field approximation and deterministic kinetics (Athenes et al 1996); this renders it necessary to introduce nuclei at the beginning of the simulations. Finally, their use of fourier transform technique also limits the applicability of their technique only to small, 2-D systems. This limitation on the system size, for example, does not allow statistical properties of the system (such as the average particle size) to be estimated and compared with those from experiments, or other theories.

\section{Conclusions}

We have presented a broad, non-exhaustive overview of computational modelling studies on microstructural evolution of misfitting precipitates; the emphasis has been on the tools employed, and their advantages and limitations. The important features of elastically stressed precipitation systems, as elucidated from these studies, have also been highlighted for the purpose of illustrating the kinds of information which can be obtained through the use of these computational techniques.

\section{Acknowledgement}

We thank the Aeronautics Research and Development Board, Government of India for funding this research programme.

\section{References}

Abinandanan T A 1991 Coarsening of elastically interacting coherent particles, Ph D Thesis, Carnegie Mellon University, Pittsburgh, USA

Abinandanan T A and Johnson W C 1993 Acta Metall. Mater. 41 17, 27

Abinandanan T A and Johnson W C 1995 Mater. Sci. Engg. B32 169

Abinandanan T A and Sankarasubramanian R 1997 Springer proc. in physics (Heidelberg: Springer Verlag) (to appear)

Abinandanan T A, Haider F and Martin G 1997 Acta Mater. (submitted)

Ardell A J 1970 Metall. Trans. 1525

Ardell A J, Nicholson R B and Eshelby J D 1966 Acta Metall. 141295

Athenes M, Bellon P, Haider F and Martin G 1996 Acta Mater. 444739

Barnett D M, Lee J K, Aaronson H I and Russell K C 1974 Scr. Metall. 81447

Binder K 1991 in Materials science and technology (ed.) P Haasen (Weinheim: VCH) Vol. 5

Born M and Huang K 1954 in Dynamical theory of crystal lattices (Oxford: Oxford University Press)

Chen L-Q and Wang Y 1996 JOM 4813

Desplat J, Bley F and Livet F 1996 Acta Mater. 442839

Eshelby J D 1957 Proc. R. Soc. A241 376

Eshelby J D 1959 Proc. R. Soc. A252 561

Fratzl P and Penrose O 1995 Acta Metall. Mater. 432921

Fratzl P and Penrose O 1996 Acta Mater. 443227 
Gunton J D, San Miguel M and Sahni P S 1983 in Phase transitions and critical phenomena (eds) C Domb and J L Lebowitz (New York: Academic Press) Vol. 8

Hort W and Johnson W C 1996 Metall. Mater. Trans. A27 1461

Johnson W C 1986 Metall. Trans. A18 233

Johnson W C and Alexander J I D 1986 J. Appl. Phys. 592735

Khachaturyan A G, Semenovskaya S V and Morris Jr J W 1988 Acta Metall. 361563

Larche F C and Cahn J W 1973 Acta Metall. 211051

Larche F C and Cahn J W 1978 Acta Metall. 261579

Leo P H and Jou H -J 1993 Acta Metall. Mater. 412271

Leo P H and Sekerka R F 1989 Acta Metall. 373119

Marro J, Bortz A B, Kalos M H and Lebowitz J L 1975 Phys. Rev. B12 2000

Miyazaki T, Nakamura K and Mori H 1979 J. Mater. Sci. 141827

Miyazaki T, Imamura H and Kozakai T 1982 Mater. Sci. Engg. 5445

Moulic A G, Sastry G V S and Lele S 1994 Bull. Mater. Sei. 17439

Mura T 1987 Micromechanics of defects in solids (Dordrecht: Martinus Nijhoff Pub). 2nd ed.

Thompson M E, Su C S and Voorhees P W 1994 Acta Metall. Mater. 422107

Tien J K and Copley S M 1971 Metall. Trans. 2215

Voorhees P W, McFadden G B, Boisvert R F and Meiron D I 1988 Acta Metall. 36207

Voorhees P W, McFadden G B and Johnson W C 1992 Acta Metall. Mater. 402979

Wang, Y, Chen L -Q and Khachaturyan A G 1991a Scr. Metall. Mater. 251387

Wang Y, Chen L -Q and Khachaturyan A G 1991b Scr. Metall. Mater. 251969 